\title{
Programación de insumos 4 bases textiles: desafios de la academia con los proveedores locales
}

Luisa María Reinoso Rojas*

\section{Resumen}

En el siguiente texto se explica por qué el abastecimiento de materias primas para el sector de la moda es de vital importancia en el desarrollo de colecciones exitosas; proceso recreado mediante los proyectos integradores de aula (PIA) en el programa Diseño de Modas de la Corporación Unificada Nacional de Educación Superior (CUN), Regional Tolima. Asimismo, se aborda el asunto de la acelerada dinámica de la circulación de insumos para los proveedores del sector moda y cómo esta propicia experiencias académicas que determinan desafíos frente al apoyo de los proveedores nacionales, locales y la academia. Entre las principales conclusiones se resalta que una comunicación adecuada entre ellos permitirá la creación de estrategias y planes de acción complementarios frente a los procesos de formación.

Palabras clave: cuerpos y creación, programación de insumos y bases textiles, proveedores, referentes locales, tecnologías

\section{Abstract}

The following text narrates how the supply of raw materials for the fashion sector is of vital importance for the development of successful collections, process recreated through the PIA Classroom Integrator Projects, in the Fashion Design program of the Corporación Unificada Nacional de Educación Superior (CUN), Regional Tolima. It also addresses how the flow of inputs has accelerated for fashion suppliers and how these processes lead to academic experiences that identify challenges to support from domestic suppliers, premises and the academy. Among the main conclusions it highlights that an adequate communication between them will allow the creation of strategies and action plans complementary to the training processes.

Keywords: bodies and creation, local referents, programming of textile and suppliers, technologies

\footnotetext{
* Diseñadora de modas, Corporación Unificada Nacional de Educación Superior (cun), Regional Tolima. Maestranda en Imagen y Relaciones Públicas, Universidad Madero, Puebla (México). Docente titular en la cun en el programa Diseño de Modas. Forma parte del equipo de investigación de la Escuela de Comunicación y Bellas Artes (Codim). Contacto: luisa_reinoso@cun.edu.co
} 


\section{Introducción}

Los proyectos integradores de aula (PIA), como parte del programa de Diseño de Modas de la Corporación Unificada Nacional de Educación Superior (CUN), Regional Tolima, establecen propuestas didácticas para la solución de problemas generados desde la academia. En general, se trata de simulacros donde el cuerpo y la tecnología son la base para los procesos de creación. Dentro de la propuesta didáctica establecida por los PIA se enmarcan una serie de parámetros por semestre académico referidos a temas de inspiración en el desarrollo de colecciones de moda. Como parte de esa directriz se tiene en cuenta la tendencia y temporada de aplicabilidad, el perfil del consumidor a quién va dirigido el producto, una carta de color, el cronograma y los porcentajes de composición de los materiales -que oscilan entre $80 \%$ naturales $^{1}$ y $20 \%$ artificiales ${ }^{2}-$.

Por medio de esta estrategia se aborda un proceso real de producción en el que el alumno atiende a un perfil de consumidor y establece una representación simbólica para fortalecer su propuesta más allá de la forma. Esta representación invita a una experiencia sensorial en la que la concepción de aquella forma o silueta no solo busca ser atractiva a la vista, sino que atiende de manera muy cercana a la idea de Schmitt (2007) según la cual el hombre recuerda el $1 \%$ de lo que palpa, el $2 \%$ de lo que oye, el $5 \%$ de lo que ve, el $15 \%$ de lo que degusta y el $35 \%$ de lo que huele con el objetivo de dar importancia al material textil y su relación con el cuerpo.
La asignatura propedéutica del ciclo técnico profesional de la carrera de Diseño de Modas en la cun Regional Tolima es Programación de Insumos y Bases Textiles. Con esta se hace una transición al nivel de profundización temático para el siguiente ciclo propedéutico y se establece como competencia del diseñador la capacidad de programar insumos ${ }^{3}$ y bases textiles ${ }^{4}$ según los estándares de calidad, tiempo y competitividad que demanda la industria. Por programación se entiende "la gestión que busca organizar las actividades que se deben llevar a cabo para cumplir con los objetivos de producción y transformación de un producto, en este caso las prendas de vestir" (Rojas, 2014, p. 23). La asignatura es fundamental para el desarrollo de los PIA: por un lado, posiciona al docente-asesor como un guía que acerca al alumno a las posibles soluciones de las problemáticas que enfrenta y, por el otro, contribuye a establecer la relación correcta entre las bases textiles y los insumos. Además, a través de esta se refuerzan las herramientas de comunicación entre la academia y el sector productivo de la moda, con el propósito de entender las interrelaciones entre el proveedor y los estudiantes, así como el papel participativo indirecto que tienen para el éxito de los PIA.

\section{Existen miles de proveedores y marcas disponibles para el comprador de moda internacional; elegir al proveedor adecuado es tan importante (si no más) como seleccionar los looks de moda que tendrán mejor venta. Contar con}

1 Las fibras naturales se obtienen a partir de fuentes orgánicas. Se dividen en fibras vegetales, compuestas por celulosa, y fibras animales, compuestas por proteínas (Udale, 2014).

2 Las fibras artificiales se fabrican a partir de fibras celulósicas (se extrae de plantas y árboles) y no celulósicas (fabricadas a partir de productos químicos) (Udale, 2014).

3 Elemento que genera un cambio económico en el proceso de fabricación de un producto o en la prestación de un servicio (Rojas, 2014)

4 Es la tela o el material donde se expone o plasma un diseño (Rojas, 2014). 
una buena cartera de proveedores y mantener una sólida relación con los mismos es un requisito indispensable para el comprador de moda profesional. (Shaw y Koumbis, 2014, p. 78)

Ante la enorme variedad de proveedores surge la importancia de encontrar un equilibrio. Se hace entonces relevante identificar una tendencia de compra y realizar una caracterización

\section{Método}

A partir de un muestreo probabilístico (al azar, simple) y un sondeo mediante la herramienta Formularios de Google, se creó un documento de recolección de información que se compartió a través del correo electrónico institucional y el Google Classroom. La muestra constó de estudiantes de primer a noveno semestre del programa de Diseño de Modas de la cun, Regional Tolima (125 estudiantes inscritos), para asegurar que todos los miembros de la población fueran incluidos. El muestreo se desarrolló a partir de un sondeo de carácter mixto con la utilización de preguntas abiertas, una de ellas con el objetivo claro de caracterizar a los proveedores que han tenido relación con los estudiantes.

Con base en el sondeo, se identificó la necesidad de un acercamiento de la academia al entorno productivo del sector moda. Como parte de la codificación se identificaron tres grandes grupos: 1) dificultad para la consecución de materiales con respecto a los criterios establecidos con los PIA; 2) la necesidad latente de innovar frente a de los proveedores que a nivel nacional y local suplen las necesidades específicas de la academia. Asimismo, es preciso promover encuentros comerciales del sector moda que logren demostrar la necesidad de la oferta y la demanda para fortalecer los vínculos y permitir la construcción de nuevos conocimientos, herramientas de comunicación y habilidades de información, elementos y destrezas que debe adquirir el estudiante para ser un comprador profesional de moda.

lo encontrado en la ciudad, y 3) adquisición de experticia y conocimiento para el entorno laboral.

Esta información fue trasmitida en la reunión docente del programa Diseño de Moda. Como plan de acción complementario a los procesos académicos se propuso la creación de una feria de proveedores para mejorar los procesos en lo que refiere a la cadena de abastecimiento, adquisición y experticia frente a compras reales e innovación de materiales con base en los requerimientos de los PIA. Se convocaron empresas con insumos genéricos con carta de color, es decir, los que la empresa suple de manera inmediata, e insumos con características específicas que hacían referencia a aquellos que iban según las condiciones del cliente y que le darán mayor valor agregado. La estrategia convocó una empresa local dedicada a insumos con características específicas (marquillas y etiquetas), una empresa nacional dedicada a insumos genéricos con carta de color y tres empresas nacionales dedicadas a bases textiles con carta de color. 


\section{Resultados}

Como proceso de identificación de la tendencia estudiantil durante el proceso de programación de insumos y bases textiles, para los PIA, como resultado del sondeo, se recibió información de la muestra aleatoria de 35 estudiantes vinculados al programa Diseño de Modas Cun, Regional Tolima (figura 1). Estos buscaron alinearse a los requerimientos de los PIA para abordar a cabalidad los parámetros exigidos.

Durante el proceso de caracterización de los proveedores se identificó, mediante la codificación de preguntas abiertas, que los más frecuentados por los estudiantes se categorizan en: quince proveedores categorizados en bases textiles y tres de insumos, que, a su vez, podemos dividir en trece empresas de carácter local y cuatro de carácter nacional.

En respuesta a esta proximidad que generan los estudiantes y su acercamiento a proveedores nacionales y locales, se desarrolló la primera feria de proveedores sector moda. Esta contó con la participación de 77 estudiantes vinculados al programa, 14 egresados y 3 proveedores (2 nacionales y 1 local).

Figura 1. Primera feria de proveedores sector moda

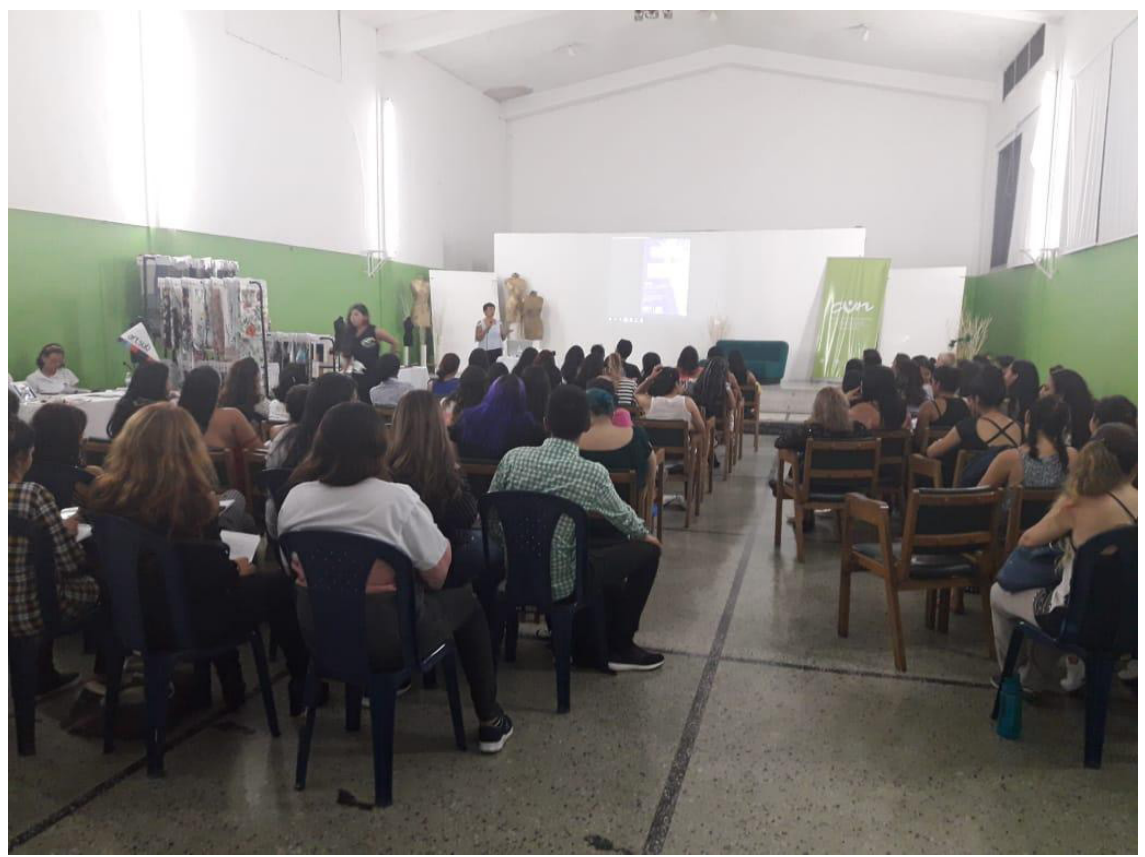

Fuente: archivo fotográfico personal de la investigadora.

\section{Discusión (o análisis de resultados)}

De acuerdo con la premisa de construcción de nuevo conocimiento, se plantea la siguiente pregunta: ¿cuál es el desafío de la academia para instruir compradores profesionales de moda? Este interrogante es aún más relevante cuando en la aplicación del método, durante la 
feria de proveedores, se realizó una encuesta de satisfacción desarrollada por un total de 38 estudiantes para determinar la probabilidad de que participarán nuevamente en una feria de este tipo. Los resultados se comparten en la figura 2.

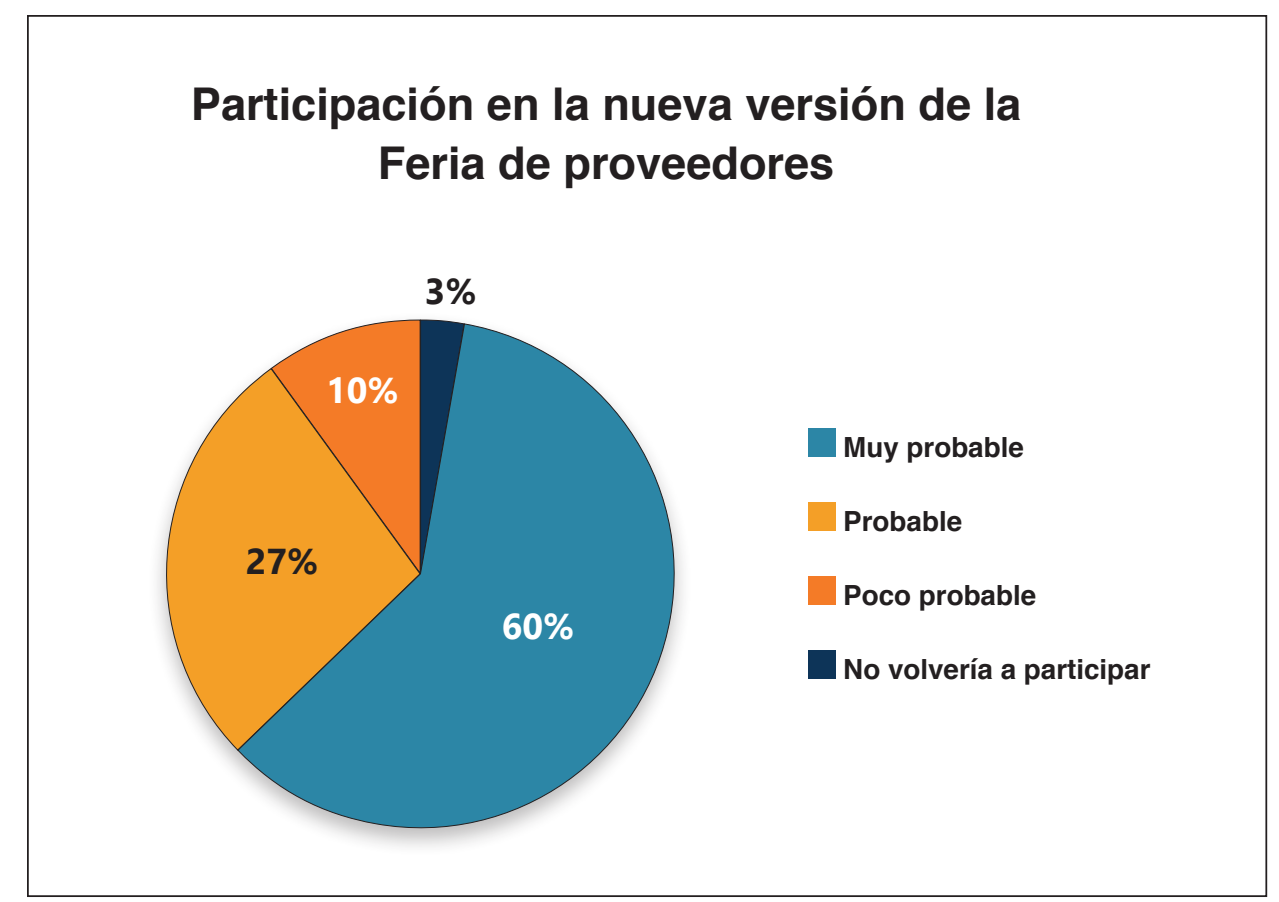

Fuente: elaboración propia.

Adicionalmente, se solicitó al parte estudiantil explicar su respuesta de manera cualitativa. A través de una codificación encontramos discrepancias frente a los resultados cualitativos. Se destacan:

1. Oposiciones frente a la logística del evento. Percepción: no cumplía con la agenda acordada.

2. Diferencias en los parámetros y procesos de compra en comparación a los solicitados por el PIA.
3. El oportuno acercamiento a proveedores y expertos para conocer los parámetros de una compra y respectivo manejo en programación de bases textiles e insumos.

En cuanto a la importancia del acercamiento a proveedores para solventar la problemática expuesta en el sondeo, al no encontrar materiales adecuados o solicitados a los proyectos integradores de aula PIA, se interactuó con los estudiantes para determinar la coherencia entre lo ofrecido por el proveedor y las solicitudes académicas (figura 3). 
Figura 3. Coherencia proveedores y PIA

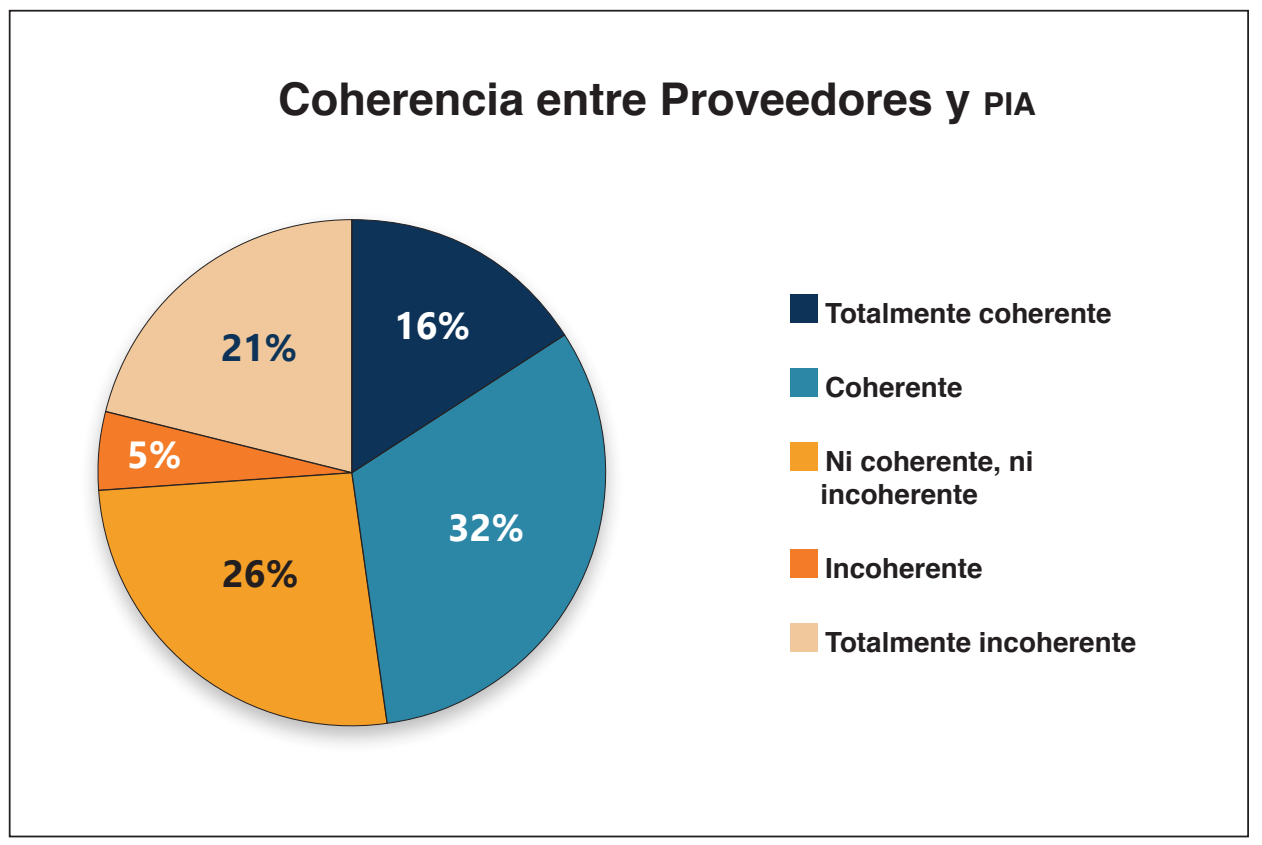

Fuente: elaboración propia.

De igual manera, se solicitó un parte cualitativo que argumentara la respuesta a la pregunta formulada.

Al codificar la información se encontraron aspectos repetitivos que proporcionaron áreas de oportunidad para incursionar desde la docencia con el objetivo de aplicar estrategias complementarias exitosas. De estas se destacan:

\section{Conclusión}

A modo de cierre, se resalta que el otorgar herramientas nuevas para el aprendizaje dirigidas a los estudiantes, con el objetivo de acercarlos a escenarios reales, es un desafío en
1. Parámetros de compra con proveedores nacionales que no estaban claros previo a la feria.

2. Percepción estudiantil de falta de investigación previa por el parte docente.

3. Exigencias de parámetros existentes a las posibilidades de compra de los estudiantes, el mínimo de compra con un proveedor nacional es de 15 metros por referencia. las aulas de clase, ya que cuando se adquieren herramientas de comunicación y se aumenta el volumen de información, se encuentran las inconsistencias en los procesos existentes. 
Tras desarrollarse la feria de proveedores, los docentes evidenciamos que algunas de las exigencias deben establecerse con base en el entorno que el sector moda nos presenta, ya sea que la exigencia se haga a nivel general sobre una base textil con la cual abarcamos la cantidad exigida por un proveedor nacional, para estipular las normativas de compra en cuanto a promedios mínimos, representantes legales, facturación y demás herramientas que intervienen en el proceso de compra.

El desafío de la academia está en incluir entre sus estrategias de formación al sector local, ya que este brinda soluciones a corto plazo en lo que a cantidades solicitadas, existencias en tiempo real y entregas inmediatas se refiere. De igual manera, se hace necesario generar vínculos económicos entre la academia y el sector moda local que permitan atender las necesidades puntuales de los actores para fomentar la innovación en el sector.

Una segunda versión del evento permitiría analizar los aspectos fallidos de la primera edición y corregirlos en pro de la generación de nuevo conocimiento, el fortalecimiento de los lazos entre la academia y el sector productivo moda, la integración de proveedores locales durante el proceso de elección de tendencias, y la consolidación de un canal de comunicación más claro, solvente y coherente con las necesidades de los perfiles del consumidor y las temporadas de usabilidad de los proyectos de aula. De esta manera, se generará un proceso óptimo para que exista un intercambio de conocimiento y se obtengan los servicios y productos requeridos.

\section{Referencias}

Rojas, N. (2014). Industria de la moda: producción y materiales. Bogotá: Ecoe Ediciones.

Schmitt, B. (2007). Experiential Marketing. Barcelona: Deusto.

Shaw, D. y Koumbis, D. (2014). La compra profesional de moda: de la predicción de tendencias al punto de venta. Barcelona: Gustavo Gilli.

Udale, J. (2014). Diseño textil: tejidos y técnicas. Barcelona: Gustavo Gilli. 